\title{
Methotrexate Dosage Reduction Upon Adalimumab Initiation: Clinical and Ultrasonographic Outcomes from the Randomized Noninferiority MUSICA Trial
}

\author{
Gurjit S. Kaeley, Amy M. Evangelisto, Midori J. Nishio, Sandra L. Goss, Shufang Liu, \\ Jasmina Kalabic, and Hartmut Kupper
}

ABSTRACT. Objective. To examine the clinical and ultrasonographic (US) outcomes of reducing methotrexate (MTX) dosage upon initiating adalimumab (ADA) in MTX-inadequate responders with moderately to severely active rheumatoid arthritis (RA).

Methods. MUSICA (NCT01185288) was a double-blind, randomized, parallel-arm study of 309 patients with RA receiving MTX $\geq 15 \mathrm{mg} /$ week for $\geq 12$ weeks before screening. Patients were randomized to high dosage (20 mg/week) or low dosage (7.5 mg/week) MTX; all patients received $40 \mathrm{mg}$ open-label ADA every other week for 24 weeks. The primary endpoint was Week 24 mean 28-joint Disease Activity Score based on C-reactive protein (DAS28-CRP) to test for noninferiority of low-dosage MTX using a 15\% margin. US images were scored using a 10-joint semiquantitative system incorporating OMERACT definitions for pathology, assessing synovial hypertrophy, vascularity, and bony erosions.

Results. Rapid improvement in clinical indices was observed in both groups after addition of ADA. The difference in mean DAS28-CRP $(0.37,95 \%$ CI $0.07-0.66)$ comparing low-dosage $(4.12,95 \%$ CI 3.88-4.34) versus high-dosage MTX $(3.75,95 \%$ CI 3.52-3.97) was statistically significant and non-inferiority was not met. Statistically significant differences were not detected for most clinical, functional, and US outcomes. Pharmacokinetic and safety profiles were similar.

Conclusion. In MUSICA, Week 24 mean DAS28-CRP, the primary endpoint, did not meet non-inferiority for the low-dosage MTX group. Although the differences between the 2 MTX dosage groups were small, our study findings did not support routine MTX reduction in MTX inadequate responders initiating ADA. (First Release June 15 2016; J Rheumatol 2016;43:1480-9; doi:10.3899/ jrheum.151009)

Key Indexing Terms:

RHEUMATOID ARTHRITIS

COMBINATION DRUG THERAPY

METHOTREXATE

ADALIMUMAB

\section{ULTRASONOGRAPHY}

\footnotetext{
From the University of Florida College of Medicine, Jacksonville, Florida; Arthritis, Rheumatic and Back Disease Associates, Voorhees, New Jersey; Diablo Clinical Research, Walnut Creek, California; AbbVie Inc., North Chicago, Illinois, USA; AbbVie Deutschland GmbH and Co. KG., Ludwigshafen, Germany.

AbbVie Inc. sponsored the study (NCT01185288), contributed to its design, participated in the collection, analysis, and interpretation of the data, and in the writing, reviewing, and approval of the final manuscript. GSK is a consultant for AbbVie Inc. AME has received research grants and served as a speaker for AbbVie Inc. SLG is an employee of AbbVie and owns stock. SL is a former employee of AbbVie and owns stock options. JK and $H K$ are full-time employees of AbbVie and own stock or stock options. G.S. Kaeley, MBBS, MRCP, University of Florida College of Medicine; A.M. Evangelisto, MD, Arthritis, Rheumatic and Back Disease Associates; M.J. Nishio, MD, Diablo Clinical Research; S.L. Goss, PhD, AbbVie Inc.; S. Liu, PhD, AbbVie Inc.; J. Kalabic, MD, AbbVie Inc.; H. Kupper, MD, AbbVie Deutschland GmbH and Co. KG.

Address correspondence to Dr. G.S. Kaeley, University of Florida College of Medicine, Division of Rheumatology, 653-1 West 8th St.,

LRC 2nd Floor L-14, Jacksonville, Florida 32209-6561, USA.

E-mail: Gurjit.Kaeley@jax.ufl.edu

Full Release Article. For details see Reprints/Permissions at jrheum.org

Accepted for publication April 19, 2016.
}

It is recommended that patients with moderately or severely active rheumatoid arthritis (RA) initiate treatment with synthetic disease-modifying antirheumatic drugs (DMARD) to prevent disease progression and inflammatory destruction of joints ${ }^{1,2}$. Methotrexate (MTX) is the most commonly prescribed first-line DMARD with proven effectiveness and safety $^{3,4}$; however, about two-thirds of patients may need more active therapy to achieve disease control ${ }^{5,6}$. Inadequate responders (IR) can enhance treatment response by adding a conventional synthetic DMARD or switching to combination treatment with a biologic, such as the anti-tumor necrosis factor (TNF) agent adalimumab (ADA) $)^{7,8}$.

While some patients achieve disease control when co-treated with ADA + MTX ${ }^{7,9,10}$, the required dosage of MTX is unclear. Whether MTX-IR patients initiating combination therapy with a TNF inhibitor require continued high-dosage MTX ( $\geq 15 \mathrm{mg} /$ week) remains unaddressed.

Another aspect of patient care is the practicality of ultrasonography (US) in assessing disease activity. Radiographic

Personal non-commercial use only. The Journal of Rheumatology Copyright $\odot$ (2016. All rights reserved 
evidence of joint disease may lag behind clinical disease ${ }^{11}$ and is not present in $70 \%$ of patients with RA at diagnosis ${ }^{12}$. US is more sensitive than conventional radiographs and clinical examinations at detecting joint changes ${ }^{13,14}$ and has detected active synovitis in patients in clinical remission or low disease activity (LDA) ${ }^{15,16}$. However, US remains a novel technique for many rheumatologists in North America. There is no international standard scoring system for quantifying or monitoring of RA disease activity by US, although validation is progressing ${ }^{11,17}$.

The purpose of the MUSICA trial was to examine the efficacy of high-dosage (20 mg/week) or low-dosage (7.5 $\mathrm{mg} /$ week) MTX in combination with ADA [40 mg every other week (eow)] in MTX-IR patients. MUSICA was the first large, randomized controlled trial to use US to assess joint disease in patients with RA over time, examining synovial hypertrophy, vascularity, and bony erosions.

\section{MATERIALS AND METHODS}

Patients. Adults $\geq 18$ years of age diagnosed with RA per the 1987 revised American College of Rheumatology (ACR) classification criteria ${ }^{18}$ were included. Patients from 47 sites in the United States and Puerto Rico had to meet the inclusion criteria and none of the exclusion criteria at the screening and baseline visits, except the requirement for $\geq 5$ tender joints out of 68 , and $\geq 5$ swollen joints out of 66 , which could be met at either visit. Patients must have had 28-joint count Disease Activity Score (DAS28) based on C-reactive protein $(\mathrm{CRP}) \geq 3.2$ at baseline, been treated with $\mathrm{MTX} \geq 15$ $\mathrm{mg} /$ week (orally and/or injectable) for $\geq 12$ weeks before screening, and discontinued all other DMARD $\geq 4$ weeks before baseline; leflunomide must have been discontinued $\geq 24$ weeks before baseline, or actively chelated with cholestyramine treatment before enrollment. Up to $10 \%$ of the patients could have used 1 prior biologic-DMARD, including abatacept, anakinra, certolizumab, etanercept (ETN), golimumab (GOL), infliximab (IFX), or tocilizumab (TCZ); otherwise, patients were biologic-naive. Patients exposed to ADA were excluded.

Study design. MUSICA (NCT01185288) was a 24-week, phase IV, multicenter, double-blind, randomized, parallel-arm study assessing the efficacy (noninferiority) of low-dosage MTX compared to maintaining high-dosage MTX treatment upon addition of ADA. The study included 2 treatment groups: $20 \mathrm{mg}$ /week oral MTX (high-dosage MTX), or $7.5 \mathrm{mg} /$ week oral MTX (low-dosage MTX). All patients received open-label (40 mg eow) subcutaneous ADA. At baseline, patients were centrally randomized 1:1 to the treatment groups and stratified by prior MTX dosage $(15,17.5$, or $\geq 20$ $\mathrm{mg} /$ week); all consented to participate in optional pharmacogenetic analysis. Only stratification of prior MTX dosage was included in the final analysis for all efficacy endpoints. Patients provided informed consent and the study was conducted in accordance with the protocol, International Conference on Harmonization, and ethical principles from the Declaration of Helsinki.

Sample size. Per historical clinical trial data with combination ADA + MTX treatment, the mean DAS28-CRP is 3.6 with SD of 1.1, and the mean change from baseline in DAS28-CRP is -2.15 with SD of 1.1 at Week 24. It was assumed that high-dosage and low-dosage MTX groups have mean DAS28-CRP of 3.6 and 3.75 at Week 24, respectively, in MUSICA. The rationale for choosing $15 \%$ as the noninferiority margin was to associate $15 \%$ of high-dosage MTX Week 24 DAS28-CRP value of 3.6 (i.e., $0.15 \times$ $3.6=0.54$ ) with a clinically acceptable diminished treatment effect of $25 \%$ of the change from the baseline (i.e., $0.25 \times 2.15=0.54$ ). With an additional assumption of SD of 1.2 for both groups, a total of 300 patients would be required to establish noninferiority with $80 \%$ power and a 2 -sided level of significance of 0.05 .
US imaging. US imaging by sponsor-trained sonographers was performed at baseline and weeks $4,8,12,16,20$, and 24 to assess inflammation at the wrist, metacarpophalangeal (MCP) joints 2, 3, 5, and metatarsophalangeal (MTP) joint 5. Images from baseline and Week 24 (or discontinued patient's last visit) were scored by 2 of 4 independent, US-experienced rheumatologists blinded to image order and treatment group; images at intervening visits were scored by 1 of 4 rheumatologists. Bilateral images based on dorsal midline imaging of the wrist, dorsal and volar imaging of MCP joints, and dorsal imaging of MTP5 were scored using a 4-grade semiquantitative scale ${ }^{19,20}$ for synovial hypertrophy and vascularity. Presence of bony erosions was assessed dichotomously using previously mentioned images in addition to lateral imaging of MCP2, medial imaging of MCP5, and plantar and lateral images of MTP5; wrists were not scored for bony erosion.

Efficacy and safety variables. Efficacy and safety outcomes were assessed, and blood samples for studies of ADA pharmacokinetics were collected before drug dosing at baseline and weeks 4, 8, 12, 16, 20, and 24. The primary endpoint was Week 24 mean DAS28-CRP. Secondary endpoints with a noninferiority margin of $15 \%$ included Week 24 proportion of patients achieving synovial vascularity improvement $\geq 30 \%$ measured by Power Doppler, ACR50, ACR70, and clinically meaningful improvement in physical function [change from baseline $\leq-0.22$ in the Health Assessment Questionnaire Disability Index (HAQ-DI)]. Exploratory analyses assessed the proportion of patients achieving clinical LDA and remission by DAS28-CRP $(<3.2$ and $<2.6$, respectively), Simplified Disease Activity Index (SDAI; $\leq 11$ and $\leq 3.3$, respectively), and Clinical Disease Activity Index (CDAI; $\leq 10$ and $\leq 2.8$, respectively). The proportion of patients achieving ACR20/50/70 response, $\triangle \mathrm{HAQ}-\mathrm{DI} \leq-0.22$, and HAQ-DI $<0.5$ were compared between treatment groups. Synovial hypertrophy, synovial vascularity, bony erosions, tender joint count (TJC), swollen joint count (SJC), physician's global assessment (PGA), and patient's global assessment (PtGA) of disease activity and pain were measured.

Adverse events (AE) were reported by system organ class and Medical Dictionary for Drug Regulatory Activities (MedDRA v15.1) preferred terms, including 18 prespecified AE attributed to MTX toxicity (described in the MTX label), assessed at each study visit.

Statistical analyses. Noninferiority was assessed using the 2-sided 95\% CI of the difference between the low-dosage and high-dosage MTX groups, based on the least squared means from the ANCOVA with baseline value, treatment group, and prior MTX dosage included in the model for continuous endpoints, and the Cochran-Haenszel-Mantel test after adjusting for prior MTX dosage for categorical endpoints. For the primary efficacy endpoint, noninferiority of low-dosage MTX could be claimed if the upper limit of the $\mathrm{CI}$ (for the difference in Week 24 mean DAS28-CRP) was less than the noninferiority margin (15\%) of the Week 24 high-dosage mean score. Last observation carried forward (LOCF) was used to account for missing data. The primary endpoint was also examined using observed data and multiple imputation for sensitivity analyses. Secondary endpoints were also examined using observed data and nonresponder imputation (NRI) for sensitivity analyses. There was no multiplicity adjustment for testing the noninferiority of the secondary endpoints. The averaged value from 2 independent, blinded readers was used to score synovial hypertrophy, synovial vascularity, and bony erosion at baseline and Week 24 , or early termination visit. The value from a single reader was used for visits at weeks 4 to 20 (data not shown).

For additional exploratory analyses, the low-dosage MTX group was assessed to determine whether it was inferior to the high-dosage MTX group in some efficacy endpoints. Safety analyses included data from all patients who received $\geq 1$ dose of the study drug. Fisher's exact test was performed for proportions of patients with AE; Poisson regression with offset of patient years (PY) was performed for number of AE per $100 \mathrm{PY}$.

\section{RESULTS}

Patient disposition. Patients with moderately to severely active RA were enrolled and randomized into 2 treatment

Personal non-commercial use only. The Journal of Rheumatology Copyright @ 2016 . All rights reserved. 
groups: low-dosage $(7.5 \mathrm{mg} /$ week, $\mathrm{n}=154)$ or high-dosage (20 mg/week, $\mathrm{n}=155$ ) MTX plus open-label ADA. Out of 393 screened subjects, 84 did not fulfill inclusion and/or met exclusion criteria. The eligible 309 patients were randomized at 1 of 47 sites (median of 6 patients per site, 1-18 patients per site). Similar numbers of patients within each treatment group completed the study, with 274/309 patients (88.7\%) overall completing 24 weeks of treatment (Figure 1). AE, withdrawn consent, and loss to followup accounted for the majority of discontinuations, and were similar in number between groups. The first patient's first visit occurred on September 13, 2010, and the last patient's last visit occurred on January 31,2013 . Mean drug compliance was $96.9 \%$ and 98.4\% for MTX and ADA, respectively.

Baseline demographics and disease characteristics. The average age of patients was 54.8 years, with mean baseline disease duration of 5.3 years and a mean duration of MTX treatment prior to the study of 1.5 years. Disease activity was high at baseline with a mean DAS28-CRP of 5.8, SDAI 42.4, and CDAI 40.9, and substantial evidence of joint disease (i.e., elevated TJC, SJC, and synovial hypertrophy scores; Table 1). Overall, the 2 treatment groups were evenly matched, with no statistically significant differences in patient demographics or disease characteristics.

Patients were MTX-IR with different prior dosages of MTX; 151 patients (48.9\%) were taking a prior stable MTX dosage of $15 \mathrm{mg} /$ week, 40 patients (12.9\%) were taking 17.5 $\mathrm{mg} /$ week, and 118 patients $(38.2 \%)$ were taking $\geq 20$ $\mathrm{mg} /$ week. Importantly, these patients were evenly randomized to the 2 treatment groups according to the stratification protocol. Prior biologic DMARD exposure was reported in 17 patients $(5.5 \%$; 7 ETN, 5 IFX, 3 GOL, 1 certolizumab, and $1 \mathrm{TCZ}$ ). Previous exposure to 1 synthetic DMARD other than MTX was reported in 25 patients $(8.1 \%) ; 5(1.6 \%)$ received 2 synthetic DMARD.

Noninferiority assessment. LOCF was used to impute for 57 of 309 patients who were missing DAS28-CRP at Week 24. After 24 weeks, the low-dosage MTX group's mean DAS28-CRP (95\% CI) was 4.12 (3.88-4.34), representing a change from baseline of $-1.67(-1.89$ to -1.45$)$. In comparison, the high-dosage MTX group's mean DAS28-CRP (95\% CI) of 3.75 (3.52-3.97) was statistically lower $(\mathrm{p}=0.014)$ and the mean change from baseline was -2.06 ( -2.28 to -1.84$)$. Although the difference of 0.37 in Week 24 mean DAS28-CRP was within the $15 \%$ noninferiority margin of 0.56 (15\% of Week 24 high-dosage mean score 3.75), noninferiority of low-dosage MTX, the primary endpoint, could not be claimed because the upper limit of the $95 \%$ CI (0.07-0.66) was greater than the noninferiority margin of 0.56 (Figure 2). Additionally, the Week $2495 \%$ CI of the difference excluded 0 .

The low-dosage MTX group was noninferior to the

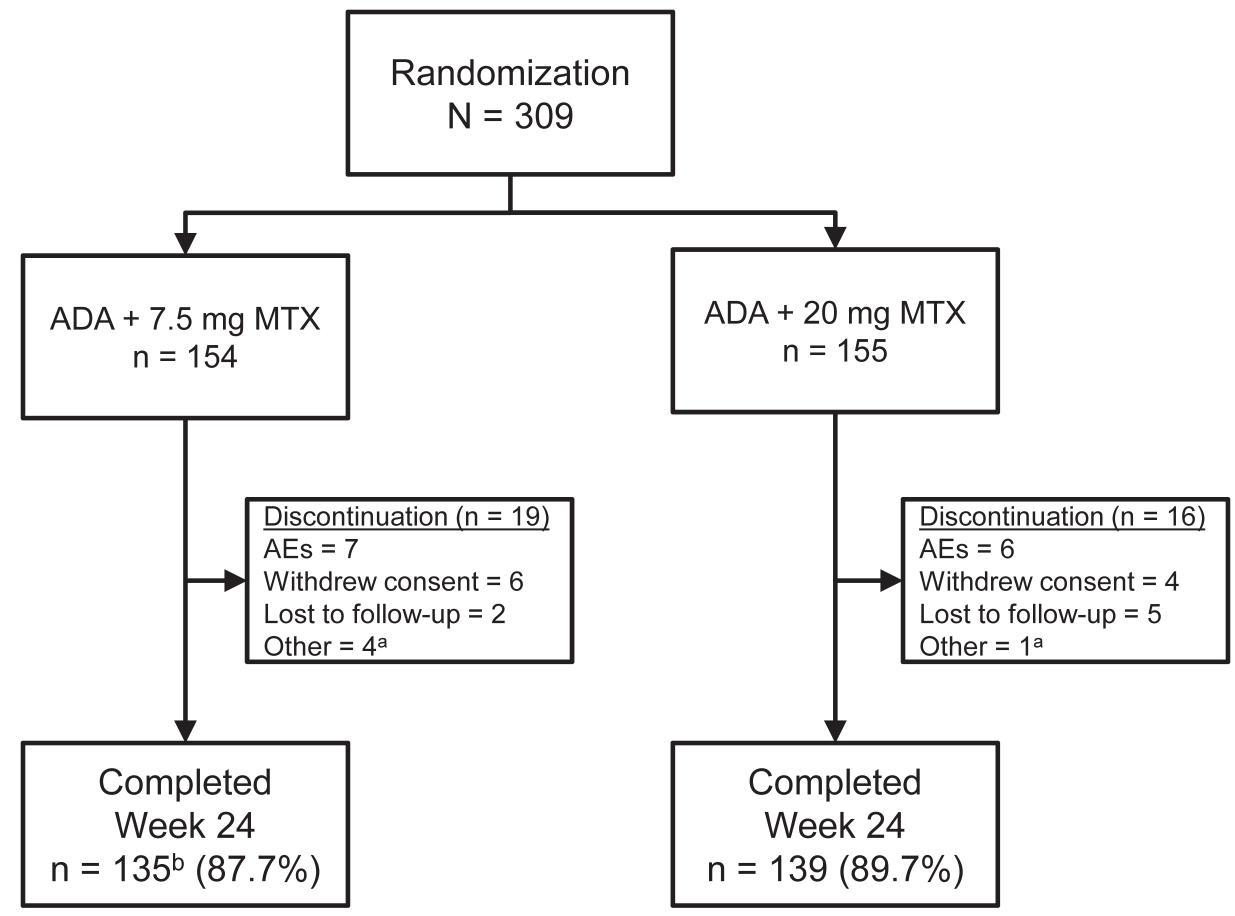

Figure 1. Patient flow. Primary reasons for discontinuation are listed. ${ }^{\mathrm{a} O n e}$ patient each with noncompliance with medications and study visits, per investigator discretion, failed inclusion criteria, and 2 patients with lack of efficacy. ${ }^{b}$ One patient delayed first dose of study drug for 2 weeks and completed the Week 24 efficacy assessment at Week 22 after first study drug dose, therefore, categorized as a Week 20 completer. ADA: adalimumab; MTX: methotrexate; AE: adverse events. 
Table 1. Patient demographics and baseline disease characteristics. Data are mean \pm SD unless indicated otherwise.

\begin{tabular}{|c|c|c|c|}
\hline Characteristics & $\begin{array}{l}\mathrm{ADA}+7.5 \mathrm{mg} \\
\mathrm{MTX}, \mathrm{n}=154\end{array}$ & $\begin{array}{l}\mathrm{ADA}+20 \mathrm{mg} \\
\mathrm{MTX}, \mathrm{n}=155\end{array}$ & Total, $\mathrm{n}=309$ \\
\hline Age, yrs & $55.1 \pm 13.1$ & $54.5 \pm 11.1$ & $54.8 \pm 12.1$ \\
\hline Age $\geq 65, \mathrm{n}(\%)$ & $37(24.0)$ & $32(20.6)$ & $69(22.3)$ \\
\hline Female, n (\%) & $120(77.9)$ & $111(71.6)$ & $231(74.8)$ \\
\hline Hispanic or Latino, n (\%) & $34(22.1)$ & $39(25.2)$ & $73(23.6)$ \\
\hline RA duration, yrs & $5.9 \pm 8.5$ & $4.7 \pm 6.5$ & $5.3 \pm 7.6$ \\
\hline \multicolumn{4}{|l|}{ Prior medication, $\mathrm{n}(\%)$} \\
\hline$\geq 1$ NSAID & $92(59.7)$ & $87(56.1)$ & $179(57.9)$ \\
\hline$\geq 1$ systemic corticosteroid & $62(40.3)$ & $67(43.2)$ & $129(41.7)$ \\
\hline Mean oral corticosteroid dose, $\mathrm{mg}$ /day & $5.4 \pm 1.8$ & $5.0 \pm 1.4$ & $5.2 \pm 1.6$ \\
\hline 1 biologic DMARD & $10(6.5)$ & $7(4.5)$ & $17(5.5)$ \\
\hline \multicolumn{4}{|l|}{ Prior MTX use, mg/week, $\mathrm{n}(\%)$} \\
\hline 15 & $76(49.4)$ & $75(48.4)$ & $151(48.9)$ \\
\hline 17.5 & $19(12.3)$ & $21(13.5)$ & $40(12.9)$ \\
\hline$\geq 20$ & $59(38.3)$ & $59(38.1)$ & $118(38.2)$ \\
\hline \multicolumn{4}{|l|}{ Duration of prior MTX use, mg/week, yrs } \\
\hline 15 & $1.8 \pm 3.3$ & $1.3 \pm 2.5$ & $1.5 \pm 2.9$ \\
\hline 17.5 & $1.1 \pm 1.3$ & $1.3 \pm 1.3$ & $1.2 \pm 1.3$ \\
\hline$\geq 20$ & $1.4 \pm 2.0$ & $1.7 \pm 3.9$ & $1.5 \pm 3.1$ \\
\hline TJC68 & $31.1 \pm 16.8$ & $31.4 \pm 17.3$ & $31.2 \pm 17.0$ \\
\hline SJC66 & $17.8 \pm 10.1$ & $18.9 \pm 11.6$ & $18.3 \pm 10.9$ \\
\hline TJC28 & $16.2 \pm 7.1$ & $16.1 \pm 7.4$ & $16.2 \pm 7.2$ \\
\hline SJC28 & $12.0 \pm 5.7$ & $12.4 \pm 5.8$ & $12.2 \pm 5.7$ \\
\hline PGA disease activity, VAS 0-100 mm & $61.6 \pm 20.6$ & $61.1 \pm 19.7$ & $61.3 \pm 20.1$ \\
\hline PtGA disease activity, VAS 0-100 mm & $62.6 \pm 22.3$ & $65.0 \pm 23.4$ & $63.8 \pm 22.8$ \\
\hline Patient pain, VAS 0-100 mm & $65.3 \pm 21.0$ & $68.3 \pm 20.8$ & $66.8 \pm 20.9$ \\
\hline $\mathrm{CRP}, \mathrm{mg} / \mathrm{l}$ & $15.4 \pm 23.6$ & $13.8 \pm 18.8$ & $14.6 \pm 21.3$ \\
\hline CRP abnormal, $\geq 9$ mg/l, n (\%) & $64(41.6)$ & $67(43.2)$ & $131(42.4)$ \\
\hline Anti-CCP, units & $1264 \pm 2393$ & $1129 \pm 2170$ & $1197 \pm 2281$ \\
\hline Anti-CCP-positive, $\geq 20$ units, $\mathrm{n}(\%)$ & $106(70.2)$ & $111(73.0)$ & $217(71.6)$ \\
\hline $\mathrm{RF}, \mathrm{IU} / \mathrm{ml}$ & $221.8 \pm 385.6$ & $175.1 \pm 348.5$ & $198.4 \pm 367.6$ \\
\hline RF positive, $\geq 12 \mathrm{IU} / \mathrm{ml}, \mathrm{n}(\%)$ & $106(69.7)$ & $104(68.0)$ & $210(68.9)$ \\
\hline DAS28-CRP & $5.8 \pm 0.92$ & $5.8 \pm 0.96$ & $5.8 \pm 0.94$ \\
\hline SDAI & $42.1 \pm 13.7$ & $42.6 \pm 13.3$ & $42.4 \pm 13.5$ \\
\hline CDAI & $40.6 \pm 12.9$ & $41.3 \pm 12.9$ & $40.9 \pm 12.9$ \\
\hline HAQ-DI & $1.45 \pm 0.59$ & $1.47 \pm 0.65$ & $1.46 \pm 0.62$ \\
\hline Bony erosion (0-22) & $1.6 \pm 2.3$ & $1.7 \pm 2.1$ & $1.6 \pm 2.2$ \\
\hline Synovial hypertrophy $(0-48)$ & $33.4 \pm 7.6$ & $34.0 \pm 7.0$ & $33.7 \pm 7.3$ \\
\hline Synovial vascularity $(0-48)$ & $5.9 \pm 5.7$ & $5.7 \pm 5.1$ & $5.8 \pm 5.4$ \\
\hline
\end{tabular}

ADA: adalimumab; MTX: methotrexate; RA: rheumatoid arthritis; NSAID: nonsteroidal antiinflammatory drugs; DMARD: disease-modifying antirheumatic drugs; TJC: tender joint count; SJC: swollen joint count; PGA: physician's global assessment; VAS: visual analog scale; PtGA: patient's global assessment; CRP: C-reactive protein; anti-CCP: anticyclic citrullinated protein; RF: rheumatoid factor; DAS28: 28-joint count Disease Activity Score; SDAI: Simplified Disease Activity Index; CDAI: Clinical Disease Activity Index; HAQ-DI: Health Assessment Questionnaire-Disability Index.

high-dosage MTX group for the proportion of patients achieving clinically meaningful improvement in physical function, a secondary endpoint (Figure 2). The additional secondary endpoints, including the proportion of patients achieving ACR50, ACR70, and synovial vascularity improvement $>30 \%$, did not meet noninferiority because the 95\% CI of the differences crossed the noninferiority margin (Figure 2).

Additional exploratory efficacy assessments. Generally, clinical disease activity measured by DAS28-CRP, SDAI, and CDAI trended together over time in the proportion of patients achieving LDA and remission based on LOCF (Figure 3). At Week 24, a numerically lower proportion of patients in the low-dosage MTX group compared to the high-dosage MTX group achieved LDA (LOCF, 28.1-32.7\% vs 35.1-38.3\%); the differences between the groups were not statistically significant at Week 24 by all 3 disease activity indices (secondary outcomes). Proportions were still increasing at Week 24. Sensitivity analyses (NRI) revealed small differences in the proportions of patients achieving LDA when comparing low-dosage (26.6-31.2\%) versus high-dosage (31.0-33.5\%) MTX groups. By LOCF, 21/153

Personal non-commercial use only. The Journal of Rheumatology Copyright (C) 2016. All rights reserved. 


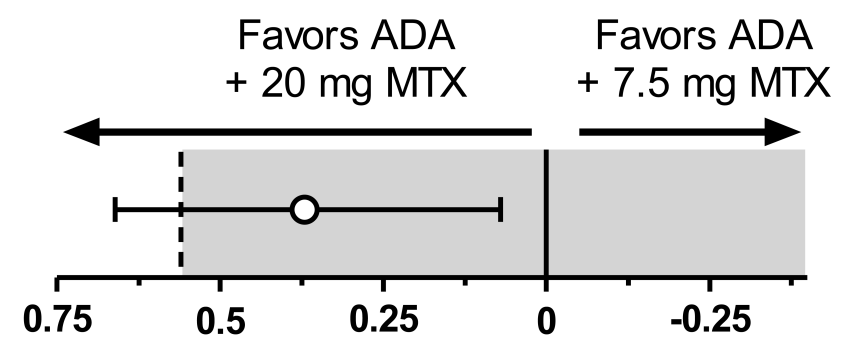

DAS28(CRP)

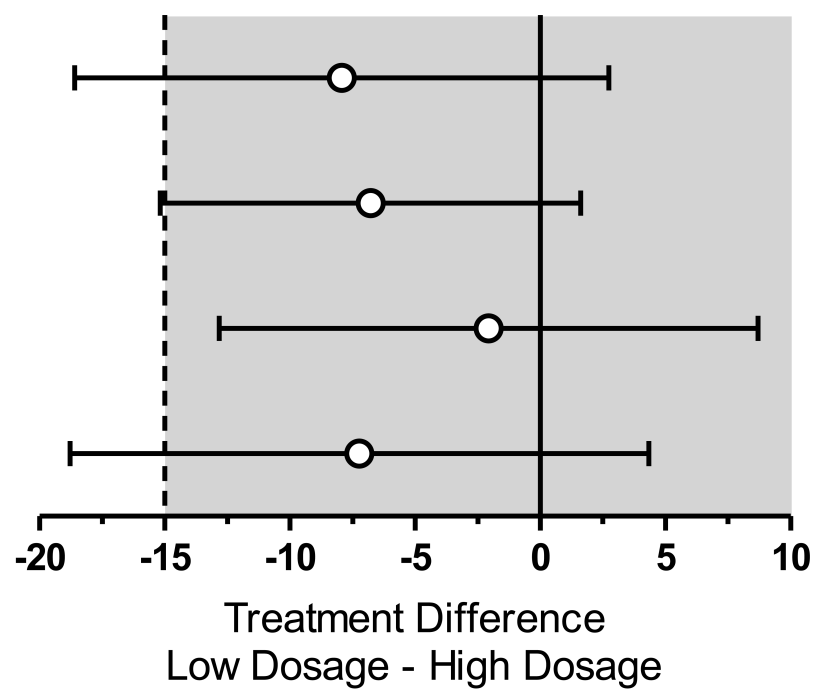

ACR50

ACR70

$\Delta \mathrm{HAQ}-\mathrm{DI} \leq-0.22$

Synovial Vascularity Improvement

Figure 2. Assessment of low-dosage MTX + ADA noninferiority compared to high-dosage MTX + ADA after 24 weeks. The difference in mean DAS28-CRP between low- and high-dosage MTX groups, and differences in proportions of patients achieving ACR50, ACR70, clinically meaningful differences in physical function, and synovial vascularity improvement (>30\%) are plotted with the difference $95 \%$ CI. The dashed line represents the noninferiority margin. ADA: adalimumab; MTX: methotrexate; DAS28: 28-joint Disease Activity Score; CRP: C-reactive protein; ACR: American College of Rheumatology; HAQ-DI: Health Assessment Questionnaire-Disability Index.

(13.7\%) and 28/154 (18.2\%) of patients in the low-dosage and high-dosage MTX groups, respectively, achieved DAS28-CRP remission $(\mathrm{p}=0.306) ; 6 / 153(3.9 \%)$ and $15 / 154$ (9.7\%) achieved SDAI remission or CDAI remission $(\mathrm{p}=0.049)$. Using sensitivity analyses (NRI) at Week 24, $21 / 154(13.6 \%)$ and 24/155 (15.5\%) in the low-dosage and high-dosage MTX groups, respectively, achieved DAS 28-CRP remission $(\mathrm{p}=0.629) ; 6 / 154(3.9 \%)$ and $13 / 155$ $(8.4 \%)$ achieved SDAI remission and CDAI remission $(p=0.100)$. Statistically significant differences in the proportion of patients remaining in high disease activity at Week 24 were observed, as measured by DAS28-CRP (26.1\% vs $16.2 \%$; $\mathrm{p}=0.036)$, SDAI $(31.4 \%$ vs $20.8 \%$; $\mathrm{p}=0.037)$, and CDAI $(35.9 \%$ vs $24.0 \% ; \mathrm{p}=0.025)$ comparing the low-dosage versus high-dosage MTX groups, respectively.

ACR responses at Week 24 numerically favored the high-dosage group. In the low-dosage and high-dosage MTX groups, respectively, $57 \%$ and $61.7 \%$ of patients achieved ACR20 ( $\mathrm{p}=0.395) ; 29.8 \%$ and $37.7 \%$ achieved ACR50 $(\mathrm{p}=0.145) ; 13.2 \%$ and $20.1 \%$ achieved ACR70 $(\mathrm{p}=0.114$;
Figure 4A). Nearly two-thirds of all patients had clinically meaningful improvement in physical function. A similar proportion of patients in the low-dosage versus high-dosage MTX group achieved $\triangle \mathrm{HAQ}-\mathrm{DI} \leq-0.22(63.6$ vs $65.6 \%$, $\mathrm{p}=0.707)$ and HAQ-DI $<0.5$ (31.4 vs $32.9 \%, \mathrm{p}=0.792$; Figure 4B). Week 24 improvements in synovial vascularity, measured by either the proportion of patients with $>30 \%$ improvement $(\mathrm{p}=0.221)$ or mean change from baseline in Power Doppler score $(\mathrm{p}=0.779)$, were not statistically different between treatment groups (Figure $4 \mathrm{C}$ and Figure 4D). By Week 24, $45.5 \%$ and $52.4 \%$ of patients in the low-dosage and high-dosage MTX groups showed an improvement in synovial vascularity (Figure 4C). Synovial inflammation and presence of bony erosions showed comparable improvements regardless of MTX dosage ( $p>0.05$; Figure 4D).

Improvements in other secondary efficacy outcomes, including TJC, SJC, PGA, PtGA, pain, and CRP were comparable between treatment groups over time (Supplementary Figure 1, available online at jrheum.org); only differences in Week 24 mean SJC and PGA were statistically significant.

Personal non-commercial use only. The Journal of Rheumatology Copyright ()$^{2016}$. All rights reserved. 
A

DAS28(CRP)

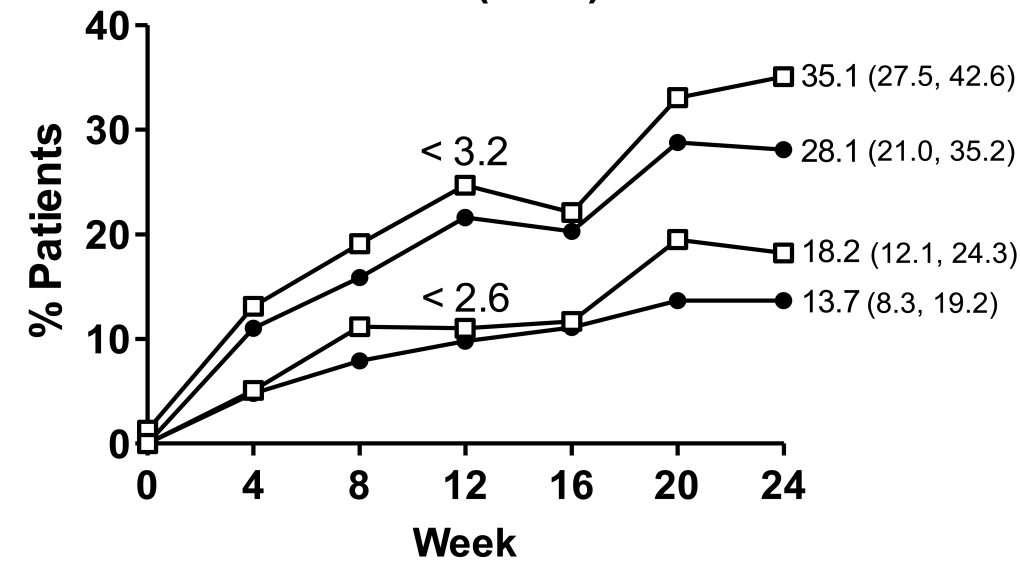

B

SDAl

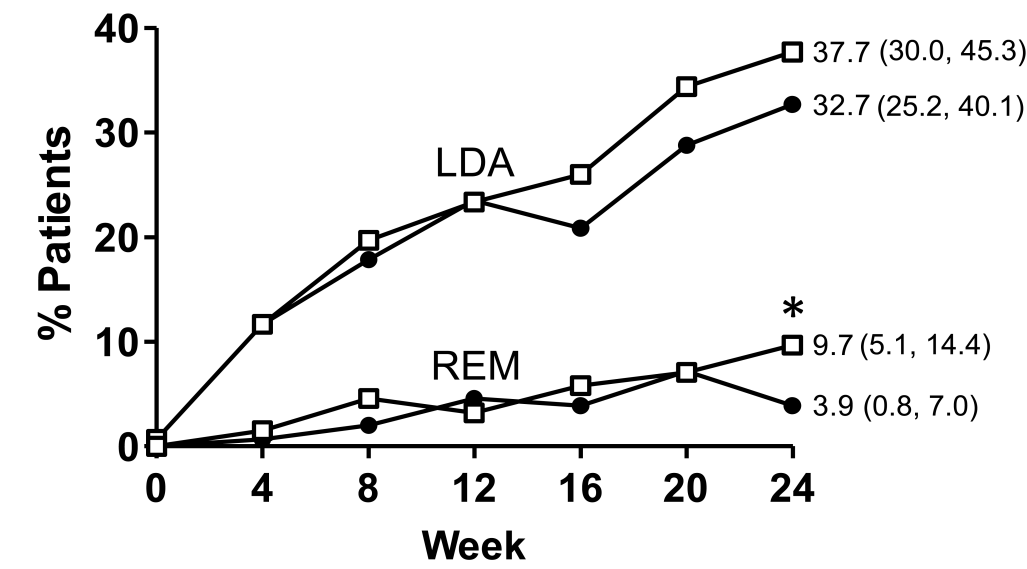

C

CDAl

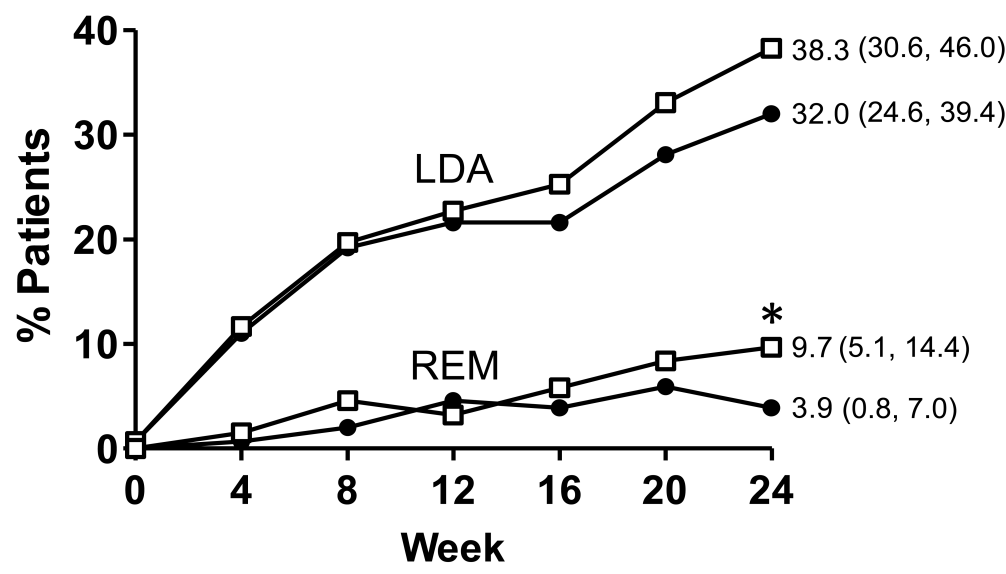

ADA + 7.5 mg MTX - $\square-A D A+20$ mg MTX

Figure 3. Clinical efficacy after 24 weeks of open-label ADA in combination with 7.5 or $20 \mathrm{mg} /$ week MTX. A. Proportion of patients over time achieving DAS28-CRP $<3.2$ and $<2.6$. B. Proportion of patients over time achieving SDAI LDA and remission. C. Proportion of patients over time achieving CDAI LDA and remission. LOCF data are presented. * Denotes statistical significance at the 0.05 level. DAS28: 28-joint Disease Activity Score; CRP: C-reactive protein; SDAI: Simplified Disease Activity Index; LDA: low disease activity; REM: remission; CDAI: Clinical Disease Activity Index; ADA: adalimumab; MTX: methotrexate; LOCF: last observation carried forward. 
A

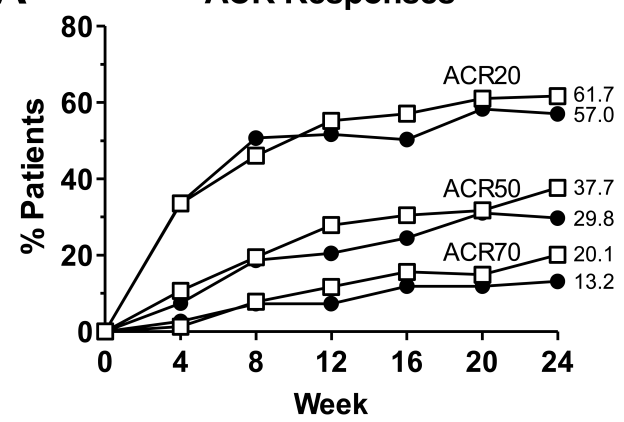

C Synovial Vascularity Improvement

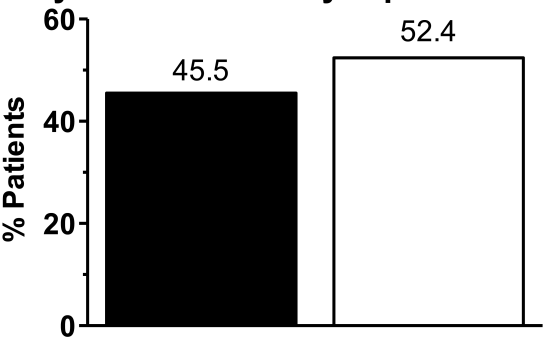

E Adalimumab Pharmacokinetics

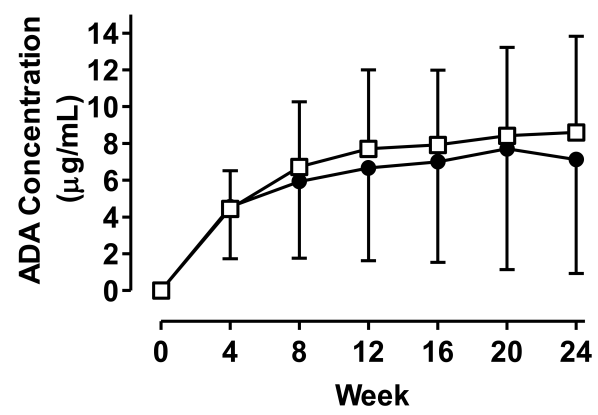

B

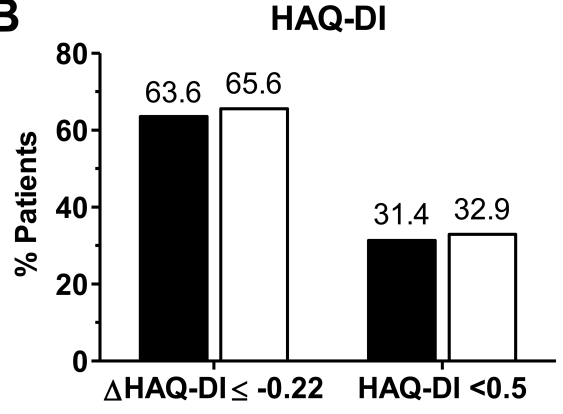

D Mean Ultrasound Improvement

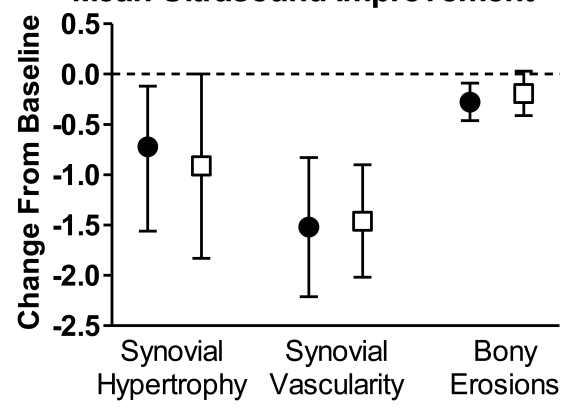

$\rightarrow$ ADA + 7.5 mg MTX

- $A D A+20 \mathrm{mg}$ MTX

Figure 4. Clinical, functional, and ultrasonographic efficacy, and ADA pharmacokinetics after 24 weeks of open-label ADA in combination with different doses of MTX. A. Proportion of patients achieving ACR20/50/70 response. B. Clinically meaningful change in physical function ( $\triangle \mathrm{HAQ}-\mathrm{DI} \leq-0.22)$ and normal function (HAQ-DI $<0.5$ ). C. Synovial vascularity improvement $>30 \%$. D. Mean changes from baseline in synovial hypertrophy, synovial vascularity, and bony erosions. E. ADA serum trough concentrations over time. Ultrasonographic scores for baseline and Week 24, or early termination visit, were the average of scores from 2 raters. ACR: American College of Rheumatology; HAQ-DI: Health Assessment Questionnaire-Disability Index; ADA: adalimumab; MTX: methotrexate.

ADA pharmacokinetics. Pharmacokinetic analyses revealed slightly higher mean ADA concentrations in the high-dosage MTX group, with separation from the low-dosage MTX group beginning at about Week 8 and maintained throughout the study (Figure 4E). Week 24 ADA mean serum concentrations for patients in the high-dosage MTX group averaged $8.6 \pm 5.2 \mu \mathrm{g} / \mathrm{ml}$ compared to $7.1 \pm 6.2 \mu \mathrm{g} / \mathrm{ml}$ for patients in the low-dosage MTX group. ADA serum concentrations observed in the treatment groups were comparable or above those observed in patients naive to MTX treatment.

Safety assessments. AE were reported by $195 / 309$ patients $(63.1 \%)$, with similar numbers in both groups. Interestingly, more events per $100 \mathrm{PY}$ were observed in the low-dosage MTX group for some AE (Table 2). Serious, severe, and infectious AE occurred in a slightly larger percentage of patients within the high-dosage MTX group; no serious infections occurred within the low-dosage MTX group. Five patients, all within the high-dosage MTX group, experienced 7 serious infections. One patient, receiving concomitant prednisone, reported 3 infections (soft tissue abscess, staphylococcal infection, and septic shock) all occurring on study Day 72; the patient remained in the study until completion. Two patients discontinued owing to serious infections: 1 experienced a moderate case of pneumonia (Day 36, 
Table 2. Safety results following 24 weeks of treatment.

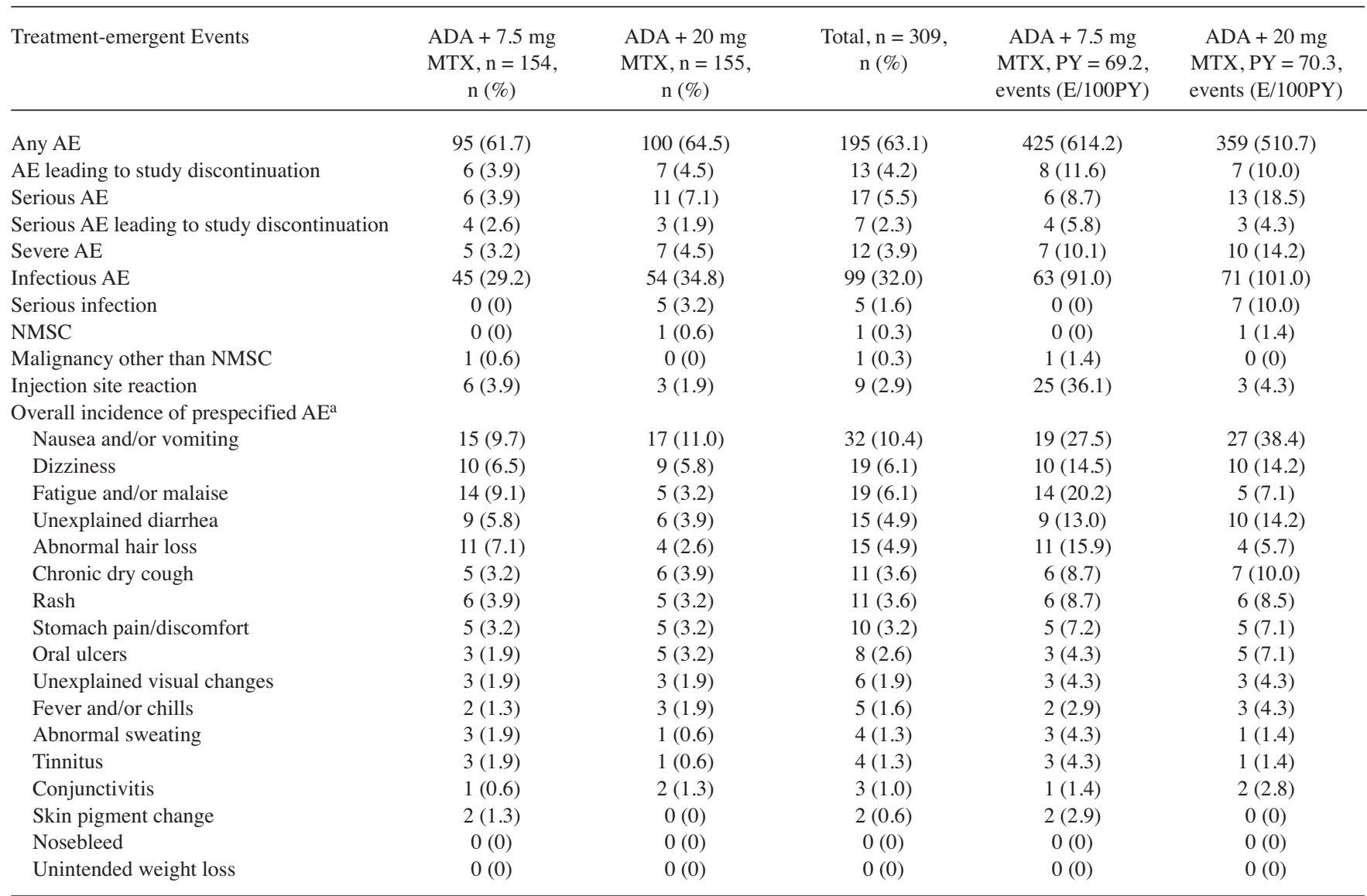

Treatment-emergent adverse event (AE) is defined as any AE with an onset date on or after the first dose of study drug, and up to 70 days after the last dose of study drug. a Assessed at each study visit for potential MTX relation. All reported AE are presented irrespective of investigator judgment. ADA: adalimumab; MTX: methotrexate; PY: patient-years; E: events; NMSC: non-melanoma skin cancer.

persisting for 22 days); another receiving concomitant prednisone experienced an ophthalmic herpes zoster infection 7 days after last dosing of study drug (Day 142) that persisted for 11 days. One patient each experienced pneumonia Legionella (Day 24, receiving concomitant prednisone) and cellulitis (Day 14); both remained in the study. Two patients reported malignancy; 1 patient in the low-dosage MTX group reported a severe bile duct cancer and 1 patient in the high-dosage MTX group reported mild squamous cell carcinoma of the skin. No new safety signals pertaining to opportunistic infections, tuberculosis, or deaths were recorded in MUSICA.

The most commonly reported AE were nausea, upper respiratory tract infection, urinary tract infection, dizziness, and headache. The only AE distinctly different between treatment groups was injection site reaction events/100 PY, which were more prevalent in the low-dosage MTX group (36.1 events/100 PY vs 4.3 events/100 PY), although all 25 events were recorded in only 6/154 low-dosage MTX patients.

Known MTX-related toxicity AE were assessed at each study visit (Table 2). Excessive fatigue and/or malaise and abnormal hair loss were interestingly more prevalent in the low-dosage MTX group (investigator-assessed). Both treatment groups had similar laboratory test and vital sign results. No new safety signals were observed.

\section{DISCUSSION}

MUSICA was the first study, to our knowledge, to investigate MTX-dosage reduction in MTX-IR patients with RA initiating biologic combination therapy, and the first randomized, blinded ADA study to assess rheumatoid-specific inflammation in patients by US. Although clinically significant improvements in disease activity were observed for both MTX dosage groups, noninferiority of low-dosage MTX + ADA was not statistically met for the primary endpoint, and a statistically significant difference of $0.37(95 \% \mathrm{CI}$ : 0.07-0.66) in mean Week 24 DAS28-CRP was observed for the low- vs high-dosage MTX group. Noninferiority of low dosage MTX + ADA was not met for 3/4 secondary endpoints. While many individual efficacy variables did not show statistically significant differences between treatment groups, they were not powered to detect differences. 
Addition of ADA to MTX-IR resulted in increased ACR responses, and a marked improvement in physical function, after 24 weeks (mean $\triangle \mathrm{HAQ}-\mathrm{DI}=-0.5$ ), similar to reports from previous ADA trials in patients with longstanding RA $^{9,21}$. Sensitivity analyses using observed data and NRI confirmed that differences between treatment groups were small, and generally smaller than when analyzed by LOCF. By US, nearly half of all patients showed improved synovial inflammation at 24 weeks compared to baseline.

Minor differences in mean ADA serum concentrations between the 2 groups were observed as early as Week 8 and up to Week $24(7.1 \pm 6.2$ and $8.6 \pm 5.2$ for the low-dosage and high-dosage MTX groups, respectively). These concentrations were consistent with the ability to achieve therapeutic response $^{22,23}$, and in agreement with DAS28-CRP and ACR responses over time. MTX is known to reduce ADA clearance by $29 \%$ and $44 \%$ after single and multiple doses, respectively ${ }^{22,24}$. The small ADA concentration difference may be due to the stability of polyglutamated $\mathrm{MTX}^{25}$ from pretrial exposure. The effect of different doses of MTX on steady-state ADA pharmacokinetics, evaluated in a parallel study of treatment-naive patients, resulted in similar ADA concentrations when co-administered with 10 or 20 mg MTX and slightly lower concentrations when co-administered with 2.5 and $5 \mathrm{mg} \mathrm{MTX}{ }^{23}$. The presence of anti-ADA antibodies (AAA) has been shown to affect ADA pharmacokinetics and efficacy $^{26}$. Levels of AAA have been demonstrated to be lower in patients receiving higher concomitant MTX dose ${ }^{27}$. In MUSICA, the overall incidence of AAA was $\operatorname{low}^{28}$ and therefore unlikely to have had a meaningful effect on ADA pharmacokinetics/efficacy.

Serious, severe, and infectious AE were slightly more prevalent in the high-dosage group. Interestingly, more $\mathrm{AE} / 100 \mathrm{PY}$ were observed in the low-dosage group, possibly as a result of disease flaring after MTX reduction. More patients in this group reported arthritis and RA as an $\mathrm{AE}^{29,30}$. Among MTX toxicity-related AE, abnormal hair loss and excessive fatigue and/or malaise were more prevalent among the low-dosage group.

The MUSICA results suggest that lowering the dose of concomitant MTX to $7.5 \mathrm{mg} /$ week from $\geq 15 \mathrm{mg}$ /week upon initiating ADA may reduce the degree of clinical response when compared to steady or slightly increased MTX. Patients were receiving $15,17.5$, or $\geq 20 \mathrm{mg} /$ week MTX prior to study entry. Patients taking prior $15 \mathrm{mg} /$ week MTX $(\mathrm{n}=151$, $48.9 \%$ ) who were randomized to the $7.5 \mathrm{mg} / \mathrm{week}$ arm had a dose reduction of $7.5 \mathrm{mg} /$ week MTX. However, patients taking higher prior MTX doses ( 17.5 to $\geq 20 \mathrm{mg} /$ week) who were randomized to the $7.5 \mathrm{mg} /$ week arm experienced a larger MTX dose reduction (10-14.5 mg/week). Patients who had a smaller reduction in MTX had better clinical responses than those who experienced a larger reduction in MTX (Supplementary Figure 2, available online at jrheum.org). The study treatment duration may limit accurate interpre- tation because the proportions of patients achieving clinical LDA and remission measured by DAS28-CRP, SDAI, and CDAI were still increasing at Week 24, suggesting that longer treatment duration may have continued to reduce disease activity. One study design limitation was the relatively large noninferiority margin of $15 \%$ for ACR50 and ACR70, which showed proportions of responders $<40 \%$ at Week 24 .

Other trials have investigated MTX dosage reduction after combination treatment or achievement of a target disease state $^{31,32}$. In the CAMEO trial, ETN monotherapy was noninferior to ETN + MTX only for patients in $\mathrm{LDA}^{31}$. In an open-label extension trial ${ }^{33}$, clinical response was maintained upon an MTX dosage-reduction (mean 17.5 to $11 \mathrm{mg} /$ week) or discontinuation, following 3 months of ETN + MTX. In MUSICA, the MTX dosage reductions upon ADA initiation were often larger, and patients had active RA. Noninferiority was not met for the mean DAS28-CRP for low- vs high-dosage MTX, and patients who decreased concomitant MTX had a slightly reduced degree of clinical response compared to those taking steady or slightly increased MTX. Overall, these data do not support MTX dosage reductions in patients initiating ADA. However, in a proportion of patients who may need to reduce MTX because of toxicity, much of the clinical efficacy of combination therapy may still be retained, especially if dosage reductions of MTX are modest.

\section{ACKNOWLEDGMENT}

Medical writing support was provided by Douglas E. Dylla, PhD, and Naina Barretto, $\mathrm{PhD}$, both full-time employees of AbbVie Inc.

\section{ONLINE SUPPLEMENT}

Supplementary data for this article are available online at jrheum.org.

\section{REFERENCES}

1. Smolen JS, Landewe R, Breedveld FC, Dougados M, Emery P, Gaujoux-Viala C, et al. EULAR recommendations for the management of rheumatoid arthritis with synthetic and biological disease-modifying antirheumatic drugs. Ann Rheum Dis 2010;69:964-75

2. Singh JA, Furst DE, Bharat A, Curtis JR, Kavanaugh AF, Kremer JM, et al. 2012 update of the 2008 American College of Rheumatology recommendations for the use of disease-modifying antirheumatic drugs and biologic agents in the treatment of rheumatoid arthritis. Arthritis Care Res 2012;64:625-39.

3. Weinblatt ME, Kremer JM, Coblyn JS, Maier AL, Helfgott SM, Morrell M, et al. Pharmacokinetics, safety, and efficacy of combination treatment with methotrexate and leflunomide in patients with active rheumatoid arthritis. Arthritis Rheum 1999;42:1322-8.

4. Hamilton RA, Kremer JM. Why intramuscular methotrexate may be more efficacious than oral dosing in patients with rheumatoid arthritis. Br J Rheumatol 1997;36:86-90.

5. van der Kooij SM, de Vries-Bouwstra JK, Goekoop-Ruiterman YP, van Zeben D, Kerstens PJ, Gerards AH, et al. Limited efficacy of conventional DMARDs after initial methotrexate failure in patients with recent onset rheumatoid arthritis treated according to the disease activity score. Ann Rheum Dis 2007;66:1356-62.

6. Emery P, Breedveld FC, Hall S, Durez P, Chang DJ, Robertson D, et

Personal non-commercial use only. The Journal of Rheumatology Copyright $\subset$ $\subset$ 2016. All rights reserved 
al. Comparison of methotrexate monotherapy with a combination of methotrexate and etanercept in active, early, moderate to severe rheumatoid arthritis (COMET): a randomised, double-blind, parallel treatment trial. Lancet 2008;372:375-82.

7. Breedveld FC, Weisman MH, Kavanaugh AF, Cohen SB, Pavelka $\mathrm{K}$, van Vollenhoven R, et al. The PREMIER study: A multicenter, randomized, double-blind clinical trial of combination therapy with adalimumab plus methotrexate versus methotrexate alone or adalimumab alone in patients with early, aggressive rheumatoid arthritis who had not had previous methotrexate treatment. Arthritis Rheum 2006;54:26-37.

8. Klareskog L, van der Heijde D, de Jager JP, Gough A, Kalden J, Malaise M, et al. Therapeutic effect of the combination of etanercept and methotrexate compared with each treatment alone in patients with rheumatoid arthritis: double-blind randomised controlled trial. Lancet 2004;363:675-81.

9. Keystone EC, Kavanaugh AF, Sharp JT, Tannenbaum H, Hua Y, Teoh LS, et al. Radiographic, clinical, and functional outcomes of treatment with adalimumab (a human anti-tumor necrosis factor monoclonal antibody) in patients with active rheumatoid arthritis receiving concomitant methotrexate therapy: a randomized, placebo-controlled, 52-week trial. Arthritis Rheum 2004;50:1400-11.

10. Kavanaugh A, Fleischmann RM, Emery P, Kupper H, Redden L, Guerette B, et al. Clinical, functional and radiographic consequences of achieving stable low disease activity and remission with adalimumab plus methotrexate or methotrexate alone in early rheumatoid arthritis: 26-week results from the randomised, controlled OPTIMA study. Ann Rheum Dis 2013;72:64-71.

11. Lopez-Ben R. Rheumatoid arthritis: ultrasound assessment of synovitis and erosions. Ultrasound Clin 2007;2:727-36.

12. Dixey J, Solymossy C, Young A. Is it possible to predict radiological damage in early rheumatoid arthritis (RA)? A report on the occurrence, progression, and prognostic factors of radiological erosions over the first 3 years in 866 patients from the Early RA Study (ERAS). J Rheumatol Suppl. 2004 Mar;69:48-54.

13. Backhaus M, Burmester GR, Sandrock D, Loreck D, Hess D, Scholz A, et al. Prospective two year follow up study comparing novel and conventional imaging procedures in patients with arthritic finger joints. Ann Rheum Dis 2002;61:895-904.

14. Baillet A, Gaujoux-Viala C, Mouterde G, Pham T, Tebib J, Saraux A, et al. Comparison of the efficacy of sonography, magnetic resonance imaging and conventional radiography for the detection of bone erosions in rheumatoid arthritis patients: a systematic review and meta-analysis. Rheumatology 2011;50:1137-47.

15. Brown AK, Quinn MA, Karim Z, Conaghan PG, Peterfy CG, Hensor E, et al. Presence of significant synovitis in rheumatoid arthritis patients with disease-modifying antirheumatic drug-induced clinical remission: evidence from an imaging study may explain structural progression. Arthritis Rheum 2006; 54:3761-73.

16. Fukae J, Isobe M, Kitano A, Henmi M, Sakamoto F, Narita A, et al Positive synovial vascularity in patients with low disease activity indicates smouldering inflammation leading to joint damage in rheumatoid arthritis: time-integrated joint inflammation estimated by synovial vascularity in each finger joint. Rheumatology 2013;52:523-8.

17. Strand V, Kingsbury SR, Woodworth T, Landewe R, Ostergaard M, Peterfy C, et al. OMERACT 10 Sharp Symposium: important findings in examination of imaging methods for measurement of joint damage in rheumatoid arthritis. J Rheumatol 2011;38:2009-13.

18. Arnett FC, Edworthy SM, Bloch DA, McShane DJ, Fries JF, Cooper NS, et al. The American Rheumatism Association 1987 revised criteria for the classification of rheumatoid arthritis. Arthritis Rheum 1988;31:315-24.
19. D'Agostino MA, Conaghan PG, Naredo E, Aegerter P, Iagnocco A, Freeston JE, et al. The OMERACT ultrasound task force advances and priorities. J Rheumatol 2009;36:1829-32.

20. Szkudlarek M, Court-Payen M, Jacobsen S, Klarlund M, Thomsen HS, Ostergaard M. Interobserver agreement in ultrasonography of the finger and toe joints in rheumatoid arthritis. Arthritis Rheum 2003;48:955-62.

21. Weinblatt ME, Keystone EC, Furst DE, Moreland LW, Weisman MH, Birbara CA, et al. Adalimumab, a fully human anti-tumor necrosis factor alpha monoclonal antibody, for the treatment of rheumatoid arthritis in patients taking concomitant methotrexate: the ARMADA trial. Arthritis Rheum 2003;48:35-45.

22. Humira. Abbott Park, IL: Abbott Laboratories; 2010

23. Burmester G, Kivitz A, Kupper H, Arulmani U, Florentinus S, Goss S, et al. Efficacy, pharmacokinetics, and safety of different doses of methotrexate in combination with adalimumab: results from the CONCERTO trial. Ann Rheum Dis 2013;72 Suppl 3:72-3.

24. Velagapudi RB, Noertersheuser PA, Awni WM, Kischkoff A, Kupper H, Granneman GR, et al. Effect of methotrexate (MTX) coadministration on the pharmacokinetics (PK) of adalimumab (HUMIRA, Abbott), following a single intravenous (iv) injection. Arthritis Rheum 2003;48:S141.

25. Dalrymple JM, Stamp LK, O’Donnell JL, Chapman PT, Zhang M, Barclay ML. Pharmacokinetics of oral methotrexate in patients with rheumatoid arthritis. Arthritis Rheum 2008;58:3299-308.

26. Bartelds GM, Krieckaert CL, Nurmohamed MT, van Schouwenburg PA, Lems WF, Twisk JW, et al. Development of antidrug antibodies against adalimumab and association with disease activity and treatment failure during long-term follow-up. JAMA 2011;305:1460-8.

27. Krieckaert CL, Nurmohamed MT, Wolbink GJ. Methotrexate reduces immunogenicity in adalimumab treated rheumatoid arthritis patients in a dose dependent manner. Ann Rheum Dis 2012;71:1914-5.

28. Ahmed G, Goss SL, Klein CE, Mozaffarian N, Kaeley GS, Awni W. Adalimumab in combination with high and low dose-methotrexate in rheumatoid arthritis patients with inadequate response to methotrexate: pharmacokinetic results from the MUSICA study [abstract]. Arthritis Rheum 2013;65 Suppl 10:S623.

29. Kremer JM, Rynes RI, Bartholomew LE. Severe flare of rheumatoid arthritis after discontinuation of long-term methotrexate therapy. Double-blind study. Am J Med 1987;82:781-6.

30. Loza E, Martinez-Lopez JA, Carmona L. A systematic review on the optimum management of the use of methotrexate in rheumatoid arthritis patients in the perioperative period to minimize perioperative morbidity and maintain disease control. Clin Exp Rheumatol 2009;27:856-62.

31. Pope JE, Haraoui B, Thorne JC, Vieira A, Poulin-Costello M, Keystone EC. The Canadian methotrexate and etanercept outcome study: a randomised trial of discontinuing versus continuing methotrexate after 6 months of etanercept and methotrexate therapy in rheumatoid arthritis. Ann Rheum Dis 2014;73:2144-51.

32. Fleischmann RM, Cohen SB, Moreland LW, Schiff M, Mease PJ, Smith DB, et al. Methotrexate dosage reduction in patients with rheumatoid arthritis beginning therapy with infliximab: the Infliximab Rheumatoid Arthritis Methotrexate Tapering (iRAMT) trial. Curr Med Res Opin 2005;21:1181-90.

33. Kremer JM, Weinblatt ME, Bankhurst AD, Bulpitt KJ, Fleischmann RM, Jackson CG, et al. Etanercept added to background methotrexate therapy in patients with rheumatoid arthritis: continued observations. Arthritis Rheum 2003;48:1493-9. 